\title{
Accurate Iris Localization Using Edge Map Generation and Adaptive Circular Hough Transform for Less Constrained Iris Images
}

\author{
Vineet Kumar, Abhijit Asati, Anu Gupta \\ Department of Electrical and Electronics Engineering, Birla Institute of Technology and Science Pilani, \\ Pilani-333031, India
}

\begin{tabular}{l} 
Article Info \\
\hline Article history: \\
Received Dec 27, 2015 \\
Revised Apr 6, 2016 \\
Accepted Apr 21, 2016 \\
\hline
\end{tabular}

Keyword:

Circular Hough transform

Edge map generation

Iris localization

Iris recognition

Iris segmentation

\begin{abstract}
This paper proposes an accurate iris localization algorithm for the iris images acquired under near infrared (NIR) illuminations and having noise due to eyelids, eyelashes, lighting reflections, non-uniform illumination, eyeglasses and eyebrow hair etc. The two main contributions in the paper are an edge map generation technique for pupil boundary detection and an adaptive circular Hough transform (CHT) algorithm for limbic boundary detection, which not only make the iris localization more accurate but faster also. The edge map for pupil boundary detection is generated on intersection (logical AND) of two binary edge maps obtained using thresholding, morphological operations and Sobel edge detection, which results in minimal false edges caused by the noise. The adaptive CHT algorithm for limbic boundary detection searches for a set of two arcs in an image instead of a full circle that counters iris-occlusions by the eyelids and eyelashes. The proposed CHT and adaptive CHT implementations for pupil and limbic boundary detection respectively use a two-dimensional accumulator array that reduces memory requirements. The proposed algorithm gives the accuracies of $99.7 \%$ and $99.38 \%$ for the challenging CASIA-Iris-Thousand (version 4.0) and CASIAIris-Lamp (version 3.0) databases respectively. The average time cost per image is $905 \mathrm{msec}$. The proposed algorithm is compared with the previous work and shows better results.
\end{abstract}

Copyright (C) 2016 Institute of Advanced Engineering and Science. All rights reserved.

\section{Corresponding Author:}

Vineet Kumar,

Department of Electrical and Electronics Engineering,

Birla Institute of Technology and Science Pilani,

Pilani-333031, India.

Email: vineet@pilani.bits-pilani.ac.in

\section{INTRODUCTION}

Iris recognition [1]-[3] is accepted as one of the most accurate biometric technologies to identify individuals and has applications in many distinct domains such as border-control services, law enforcement, secure transactions and payments, customer authentication, social-media forums, smart devices, privacy and data protection etc. The iris segmentation is an important stage in an iris recognition system, which mainly deals with localizing iris's inner and outer boundaries (i.e. iris localization) in the captured iris image. The highly accurate iris recognition systems demand for the iris images captured under constrained imaging environments and with subjects' full cooperation [4]. However, this restricts the range of domains where the iris recognition can be applied. The iris localization with high accuracy can be achieved in the constrained iris recognition systems, but it is challenging to get accurate iris localization in the less constrained systems.

The less constrained (noisy) iris images (Figure 1(b)) may contain reflections caused by a light source and non-uniform illumination caused by the position and angle of the light source while acquiring the 
images. The other non-ideal issues in the noisy iris images are heavy iris-occlusions by the eyelids and eyelashes, eyeglasses, low contrast, and eyebrow hair etc. [5]. Moreover, the iris images may have nonfrontal view when user is not looking ahead towards the camera. The iris images captured using near infrared (NIR) illuminators are preferred over visible wavelength (VW) images as their irises reveal rich and complex features [2],[6]. Therefore, most of the standard iris databases available on the internet are the NIR images [4]. Figure 1 shows the sample images from two different NIR databases; where Figure 1(a) is a more closeup iris image as compared to Figure 1(b). It is easier to localize iris in Figure 1(a) as it has bigger pupil and iris regions with a lesser surrounding area, as compared to Figure 1(b).

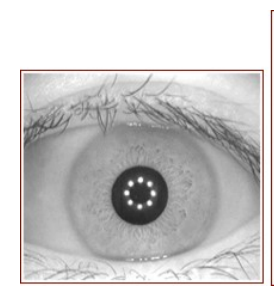

(a)

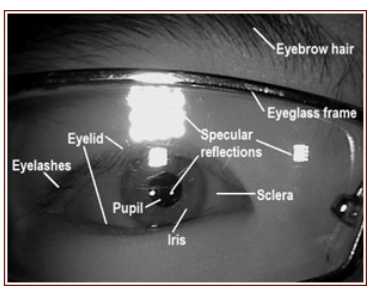

(b)

Figure 1. (a) Iris image from CASIA-Iris-Interval, version 3.0; (b) Less constrained iris image from CASIAIris-Thousand, version 4.0

The earlier iris recognition systems are typically based on Daugman's [1] and Wildes' [2] algorithms, which use integro-differential operator (IDO) and circular Hough transform (CHT) respectively to localize irises. However, their iris localization algorithms work under very controlled environments and they do not perform very accurately while dealing with the noisy images [6]. Some recent methods to localize irises in noisy NIR and VW images are described in [5],[7]-[9] and [10]-[12] respectively. Hough transform (HT) based iris localization algorithms consider the iris as a circular ring and the CHT is used to detect the circles as illustrated in [7],[10],[12].

The literature review reveals that the existing iris localization algorithms for the NIR images detect the pupil using either intensity thresholding [13],[14] or edge detection based segmentation techniques [7],[15],[16]. In the CHT based algorithms, first optimal edge maps of the iris image are generated that contain minimal false edges, so that the iris circles can be detected accurately and efficiently as demonstrated in [7] and [15]. The generating optimal edge maps get more challenging if the images are noisy such as CASIA-Iris-Thousand, version 4.0 (CITHV4) database [17] images. The noisy images are first preprocessed to remove the noise such as lighting reflections, non-uniform illumination and low contrast as described in [6]-[9], which improves the accuracy and time performance of the iris localization. The image inpainting techniques are used for removing the lighting reflection spots of the iris images and the histogram equalization is used for compensating the non-uniform illumination and low contrast [7]. For the iris localization in noisy NIR images from CITHV4 database, Wang et al. [7] proposed an inpainting technique based on Navier-Stokes equations to remove the lighting reflection spots and Probable boundary $(\mathrm{Pb})$ edge detection operator to counter the non-uniform illumination.

In this paper, the proposed iris localization algorithm has advantages that it eliminates the image preprocessing steps such as inpainting to remove reflections and methods to compensate non-uniform illumination, but still reduces the false edges caused by different types of noise very significantly. In the proposed algorithm, the edge map for pupil boundary detection is obtained by combining two different edge maps using intersection operation on images, whereas the previous iris localization methods in the literature are not based on combining two or more edge maps in a single edge map. Having detected the pupil boundary using CHT, the proposed adaptive CHT is used to detect the limbic boundary (iris's outer boundary). The proposed adaptive CHT detects arcs in the image as the eyelids and eyelashes occlude the limbic boundary, whereas the previous CHT based iris localization methods search for a full circle. The proposed algorithm targets frontal view, but noisy NIR images (Figure 1(b)) having non-ideal issues as discussed before. To evaluate the performance of the proposed algorithm, the challenging CITHV4 and CASIA-Iris-Lamp, ver. 3.0 (CILV3) iris databases [17] were used. The objective of the work presented in this paper is to overcome the constraints in achieving highly accurate biometric iris recognition.

The rest of the paper is organized as follows. Section 2 describes the proposed algorithm and its implementation, whereas section 3 discusses the performance evaluation results and the comparison with other methods. Section 4 concludes the work in the paper. 


\section{THE PROPOSED IRIS LOCALIZATION ALGORITHM}

The proposed algorithm achieves iris localization for the NIR images in two phases: Phase 1) Pupil boundary detection, and Phase 2) Limbic boundary detection. The each phase consists of two process steps, which are edge map generation from iris image and circle detection in the edge map. The goal of the edge map generation is to prepare appropriate input for CHT so that the iris circles can be detected accurately and rapidly. The original iris image of size $640 \times 480$ pixels is scaled down to $320 \times 240$ pixels using a scaling factor of 0.5 to speed up the processing. The proposed algorithm is applied on the scaled iris image and the obtained circle's parameters are multiplied by two for mapping the parameters in the original iris image.

\subsection{Phase 1: Pupil boundary detection}

The two steps involved in the pupil boundary detection are the edge map generation and the CHT for pupil boundary detection, which are discussed below.

\subsubsection{Edge map generation}

The idea of generating an optimal edge map for pupil boundary detection relies on combining two edge maps obtained via two paths: Path 1 is applying intensity thresholding on the iris image to segment the pupil region followed by the edge detection; and Path 2 is applying the edge detection on the intensity iris image. Since both the edge maps obtained via Path 1 and Path 2 have pupil contour in common, they are combined in a single edge map using the intersection operation (logical AND), which minimizes the false edges due to noise such as eyelids, eyelashes and lighting reflections etc. significantly. The proposed edge map generation is illustrated with help of Figure 2 and Figure 3. The edge map in Figure 2(e) obtained via Path 1 excludes the effect of reflections, but contains the edges due to dark illumination, whereas the edge map in Figure 2(f) obtained using Path 2 excludes the edges due to dark illumination, but contains the edges due to reflections. Therefore, the intersection operation on the two edge maps (Figure 2(e) and Figure 2(f)) removes the effect of both reflections and dark illumination as shown in Figure 2(g). To get more advantage out of the intersection operation in reducing the false edges, the two morphological operations are also used in Path 1.

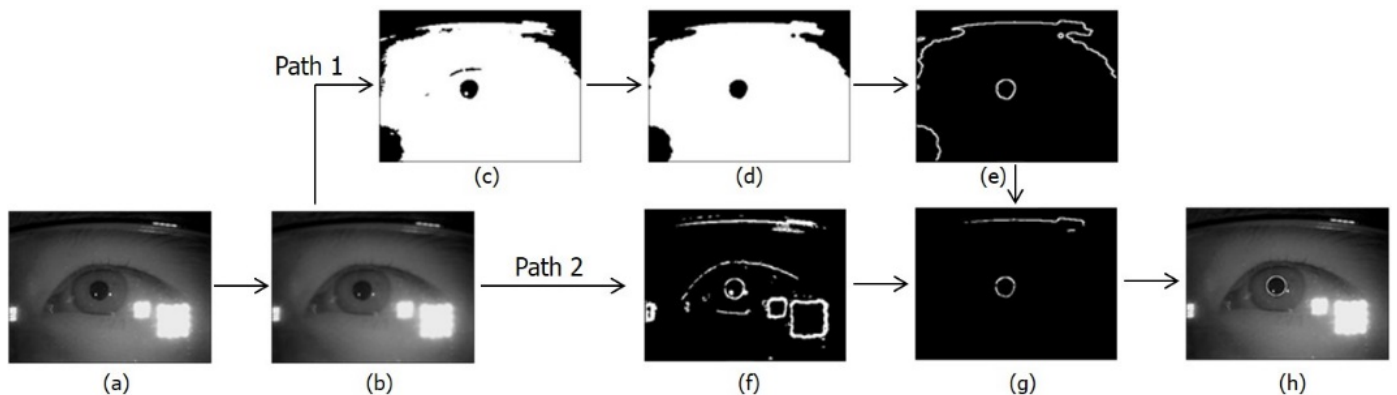

Figure 2. Edge map generation for pupil boundary detection: (a) Iris image $(320 \times 240)$ from CITHV4; (b) Gaussian smoothed iris image $(\sigma=1.0, \mathrm{k}=5)$; (c) Binary image after applying intensity thresholding on (b); (d) Cleaned binary image obtained from (c) using hole filling followed by image opening ( $\mathrm{se}=$ 'disk', $\mathrm{k}=7$ ); (e) Edge image obtained after applying Sobel edge detector without thinning on (d); (f) Edge image obtained after applying Sobel edge detector without thinning on (b); (g) Edge map obtained by intersection (logical AND) operation on (e) and (f); (h) Iris image with pupil localization (shown by white circle) obtained after applying CHT on (g)

The two morphological operations are applied on the binary image in Figure 2(c) to get the cleaned binary image shown in Figure 2(d); and the objective of these operations is reducing the noise-size so that the noise edges can be avoided in the intersection operation, which is illustrated later using Figure 3. First, a hole filling operation is applied on the binary image in Figure 2(c) to fill the white dots in the pupil region and then the image opening operation for black objects using a structuring element of type disc [18] is applied to reduce the size of the noise due to eyelids, eyelashes and eyebrow etc. Figure 2(d) shows the cleaned binary image in which the noise due to eyelids and eyelashes has been completely removed, but if the noise doesn't remove completely, its size reduces because the black regions of eyelids along with eyelashes in the binary image are not solid boundary compact objects like the pupil and the image opening operation removes the pixels at their boundaries. 

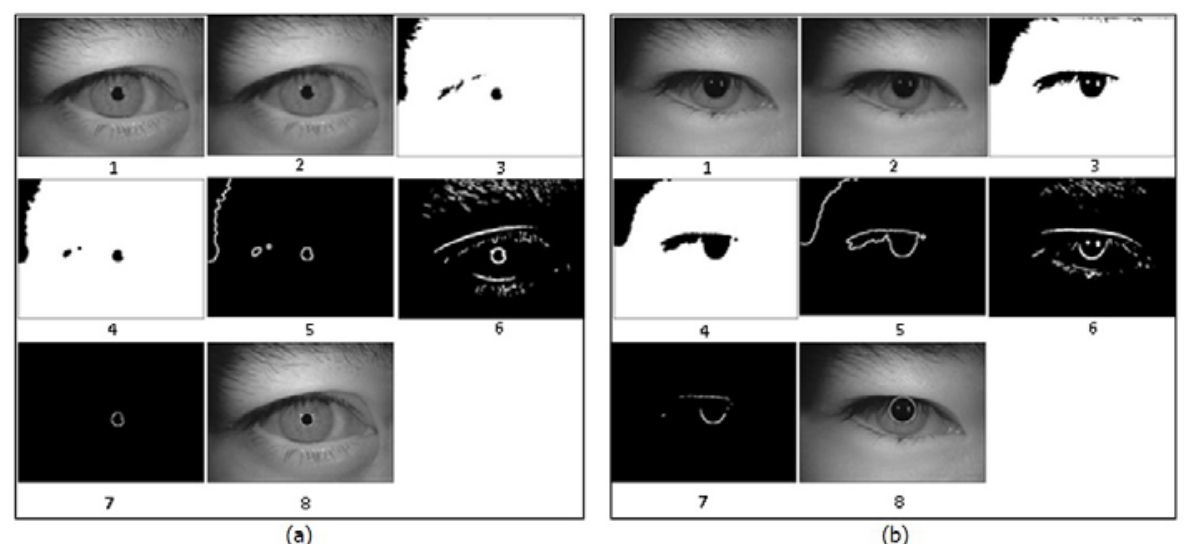

Figure 3. Edge map generation for pupil boundary detection: (a) Ideal edge map (image 7) that contains pupil boundary edges only; (b) Edge map (image 7) that contains pupil boundary edges as well as false edges; the images in (a) and (b) are: 1. Iris image from CILV3; 2. Smoothed iris image; 3. Binary image after thresholding $2 ; 4$. Cleaned binary image obtained from $3 ; 5$. Edge image of $4 ; 6$. Edge image of $2 ; 7$. Edge map obtained by intersection operation on 5 and $6 ; 8$. Pupil localized iris image obtained after applying CHT on 7.

The edge image of the cleaned binary image, shown in Figure 2(e), has the false edges due to dark illumination and eyeglass frame, but it could have contained other false edges due to the eyelids and eyelashes that can be removed or minimized by the intersection operation as illustrated using Figure 3 .

Figure 3 shows that the edge image of the cleaned binary image (image 5) contains the false edges due to eyelids and eyelashes, but these false edges are removed completely or partially after the intersection operation as shown in image 7 . The image opening operation on the binary image (image 3 ) reduces the size of the noise due to the eyelids and eyelashes and hence, the reduced noise-size in the cleaned binary image (image 4 ) is not same as detected by the edge detection on the original iris image (image 6). Therefore, the intersection operation on the image 5 and the image 6 avoids the noise-edges completely or partially. Figure 3(a) shows an ideal situation, where the intersection operation removes the false edges completely (image 7), but the edge map in Figure 3(b) has a few false edges also (image 7).

\subsubsection{CHT for pupil boundary detection}

There are a number of different approaches that can be taken in the CHT implementation [18]-[20]. To meet the requirement of detecting a circle in the edge map of iris image, we propose an implementation technique for CHT that detects a single strongest circle in an image. The proposed CHT implementation described in Algorithm 1 uses a 2-D accumulator to store votes for one radius at a time, whereas the standard CHT requires a 3-D accumulator to store votes for multiple radii that results in large storage requirements and long processing times [20]. At all the edge pixels $(\mathrm{a}, \mathrm{b})$, which are the white pixels in the edge map, the virtual circles are drawn with different radii using Equation (1). A circle with radius $r$ and center $(a, b)$ can be described with parametric equations below.

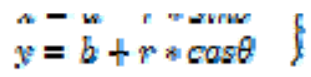

When angle $\theta$ sweeps by full 360 degrees, the circle-points (x,y) lying on the perimeter of the circle are generated. A 2-D accumulator array of size same as the image is initialized to zero. The cells' values in the array are incremented by one every time a circle passes through the cells; the process is known as accumulator voting as shown in Algorithm 1. The peak (maximum value) in the 2-D accumulator array is determined for every radius. The maximum among all the peaks gives center and radius of the detected circle. The 2-D accumulator array after voting is shown in Fig. 4 when the CHT is applied on the edge map of Figure 2(g). In Figure 4, the radius (r) is equal to the pupil radius; therefore, the coordinates of the peak in the 2-D accumulator array are the coordinates of the pupil center. 
Algorithm 1. CHT for pupil boundary detection using 2-D accumulator array

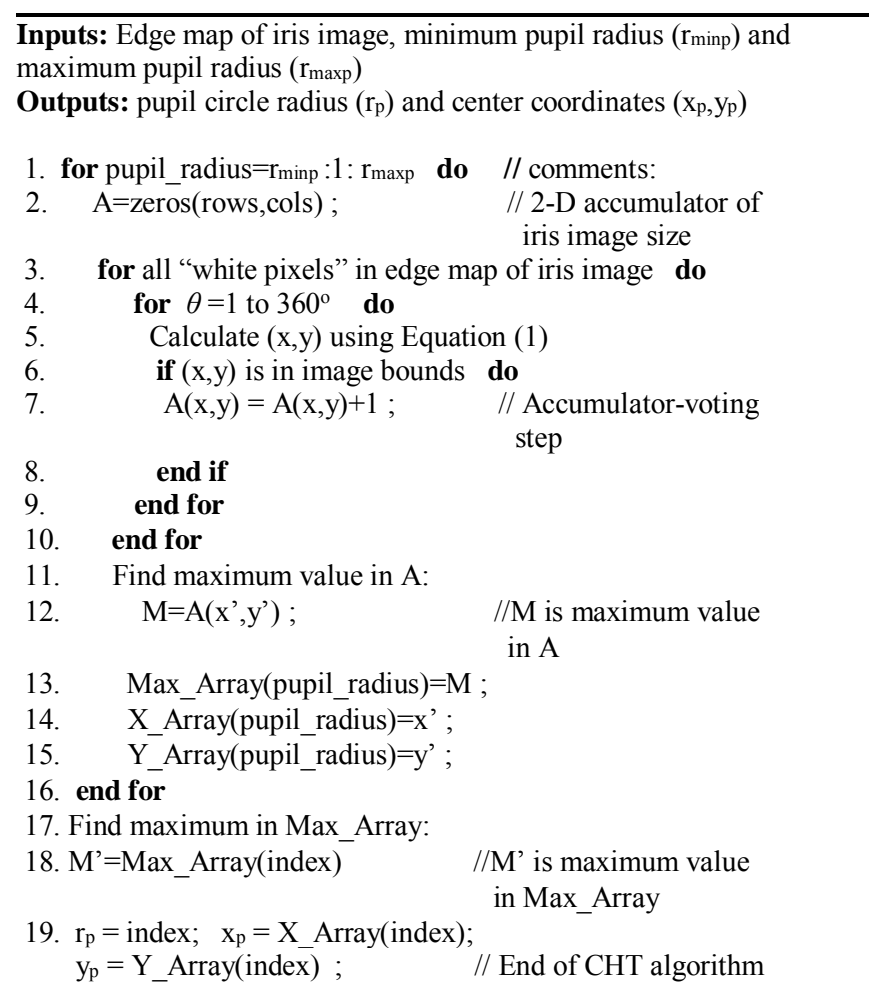

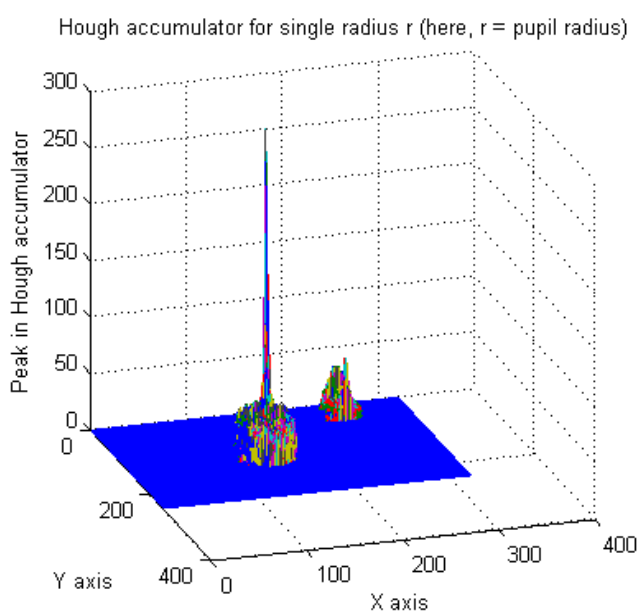

Figure 4. The surface plot of the 2-D accumulator array after voting is over corresponding to one radius

\subsection{Phase 2: Limbic boundary detection}

The center of the pupil circle is used as an input in detecting the limbic boundary as shown in Figure 5. The edge map generation and adaptive CHT for limbic boundary detection are discussed below.

\subsubsection{Edge map generation}

The limbic boundary detection may be hurdled by the eyelids, eyelashes, reflections and low contrast between the iris and sclera in the iris images [5]. A subimage is extracted from the iris image using a rectangle centered at the pupil center as shown in Figure 5(a) and Figure 5(b). The width of the rectangle (or subimage) is twice the maximum possible value of the limbic boundary radius and the height is half of the width. The height of the rectangle can be increased further if the iris-occlusion by the eyelids and eyelashes is not much. The size of the rectangle remains constant for all the images from a database, but the location of the rectangle in the image changes as the rectangle is positioned using the pupil center.

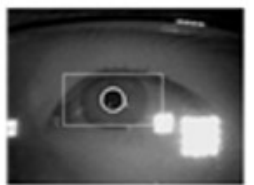

(a)

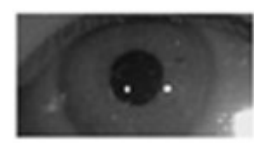

(b)

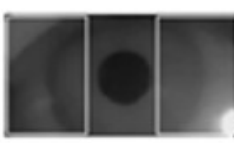

(c)

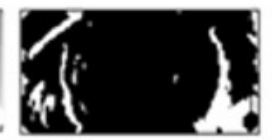

(d)

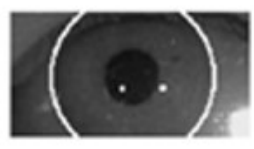

(e)

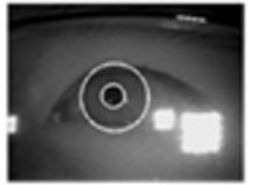

(f)

Figure 5. Limbic boundary detection: (a) Iris image $(320 \times 240)$ after pupil boundary detection; the rectangle in white indicates the size of subimage to be processed for limbic boundary detection; (b) The subimage $(130 \times 65)$ extracted from the iris image using the rectangle in (a); (c) Filtered subimage after applying a median filter of size $9 \times 9$ on (b); the two rectangles in white on left and right sides of the pupil are used to cover the iris's vertical contours; (d) Edge map obtained after applying Sobel edge detection without thinning in horizontal direction inside the two rectangles in (c); (e) Circle detection after applying the adaptive CHT on (d); (f) Iris localized image $(320 \times 240)$ 
The subimage in Figure 5(b) is filtered using a median filter [21] to suppress the noise such as the eyelash hair and uneven pixel intensities without damaging the edge structure. The upper and/or lower eyelids occlude the iris in the noisy iris images, but the vertical iris contours are always visible, which are used for detecting the limbic boundary. The vertical iris contours are covered using two rectangles that are placed as shown in Figure 5(c). The three sides of each rectangle touch the subimage borders and the fourth side of each rectangle is at a distance of pupil radius $\left(r_{p}\right)+5$ from the pupil center. To get the edge pixels, the Sobel edge detection without thinning operation is applied in the two rectangles in horizontal (x) direction only. Figure 5(d) shows the edge pixels that are used for the limbic boundary detection using the proposed adaptive CHT.

\subsubsection{Adaptive CHT for limbic boundary detection}

A. Radman et al. [22] had proposed an adaptive IDO for the limbic boundary detection, but here, we propose an adaptive CHT for the limbic boundary detection. Instead of using the general CHT algorithm for the circle detection [20], an adaptive CHT for the circular arc detection is applied on the edge map shown in Figure 5(d). The adaptive CHT detects a structure of two circular arcs defined by $-45: 45$ and 135:225 degree as shown in solid in Figure 6. The voting space in the adaptive CHT is limited to a small region around the pupil center instead of the whole image. The adaptive CHT for limbic boundary detection is useful for the images having iris-occlusions by the eyelids and eyelashes.

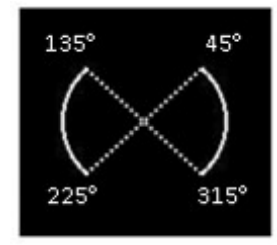

Figure 6. A set of two vertical arcs that the adaptive CHT finds in an image

The accumulator voting part of the adaptive CHT for limbic boundary detection is described in Algorithm 2. At all the white pixels $(a, b)$ in the edge map, the arcs' structure shown in Figure 6 is drawn using the Equation (1) for a radius (r) and corresponding voting is done. The size of the 2-D accumulator is same as the subimage, but voting space in the accumulator is limited to a $10 \times 10$ rectangle centered at the pupil center because the centers of the pupil and limbic boundary circles lie within a small window [6]. The peak in the 2-D accumulator is determined corresponding to each radius and the maximum among the peaks gives the center and the radius of the limbic boundary circle. The 2-D accumulator after voting is shown in Figure 7 when the adaptive CHT is applied on the edge map of Figure 5(d). The Figure 7 shows the surface plot of the 2-D accumulator corresponding to a radius equal to the limbic boundary radius and hence, the coordinates of the peak in the accumulator are the center coordinates of the limbic boundary circle. The adaptive CHT for limbic boundary detection is faster also as it searches for half the circle length instead of a full circle, which requires only half the virtual circle length to be drawn at each edge pixel. 
Algorithm 2. HT accumulator voting in adaptive CHT for limbic boundary detection

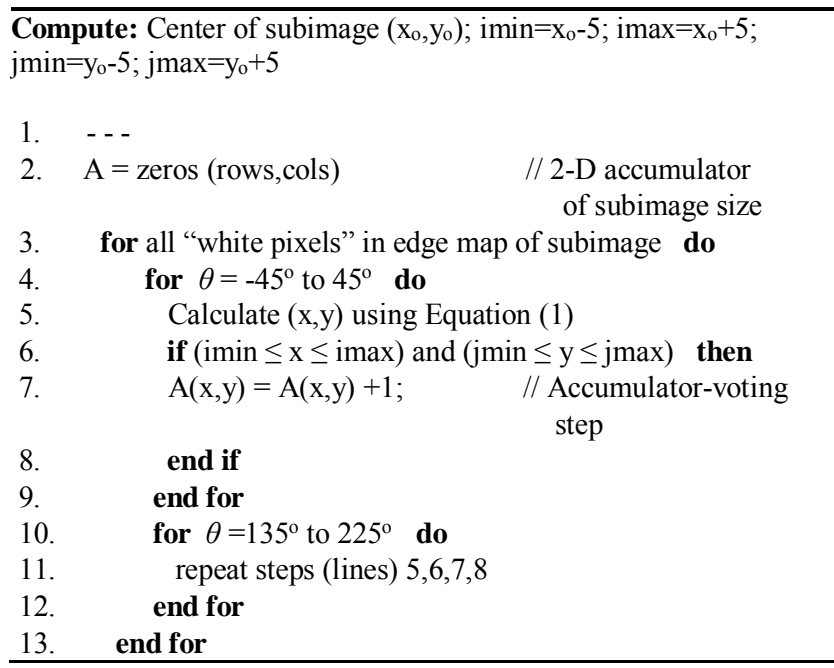

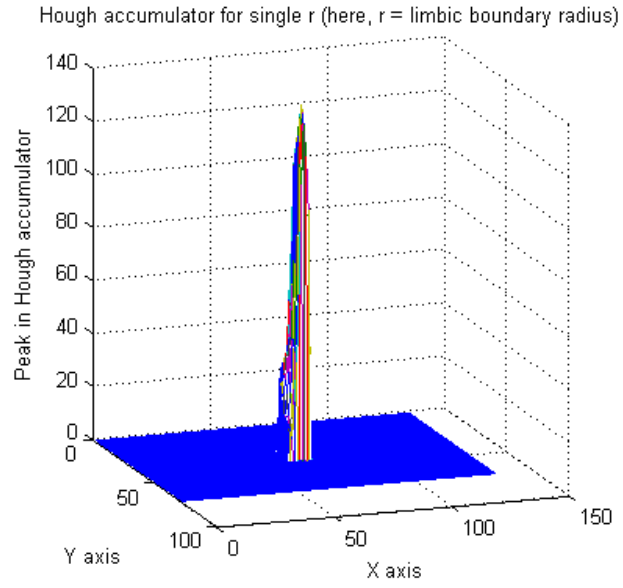

Figure 7. The surface plot of the 2-D accumulator array in the adaptive CHT after voting is over corresponding to one radius; note that voting space is a $10 \times 10$ rectangle centered at pupil center

\section{PERFORMANCE EVALUATION}

In this section, the performance of the proposed algorithm is evaluated by conducting experiments on CASIA iris databases, the iris localization results are summarized and the results are compared with some state-of-the-art iris localization methods in the literature. The datasets used in the experiments to evaluate the proposed algorithm are described below.

\subsection{Datasets used}

The datasets are taken from two CASIA iris databases [17]: CITHV4 and CILV3. These databases are chosen because they contain the noisy images having the noise such as reflections, non-uniform illuminations, low contrast, eyeglasses and intrusions by the eyelids, eyelashes and eyebrow hair. Both CITHV4 and CILV3 contain 8-bit gray-level JPEG images with resolution of $640 \times 480$ pixels.

- CITHV4 dataset: The total number of images in this database is 20000 collected from 1000 different subjects [17]. Each subject contributes 20 images, which include 10 images from each left and right eye. For extensive experimentation with this database, the images from all 1000 subjects are chosen. A total 5600 images are chosen which include all the images of the first 100 subjects and 3600 images from the rest of 900 different subjects (selecting 4 images from each subject).

- CILV3 dataset: This database contains images from 411 different subjects [17]. The total number of the images in the database is 16212. For thorough experimentation with the database, 811 images were chosen selecting first left and first right eye image of each subject except 11 subjects.

The experiments on the datasets were done using a computer with Intel i5 CPU@2.40 GHz, 8 GB RAM and Windows 7 operating system. The proposed algorithm is implemented and tested with MATLAB (version 8.4) tool.

(a)
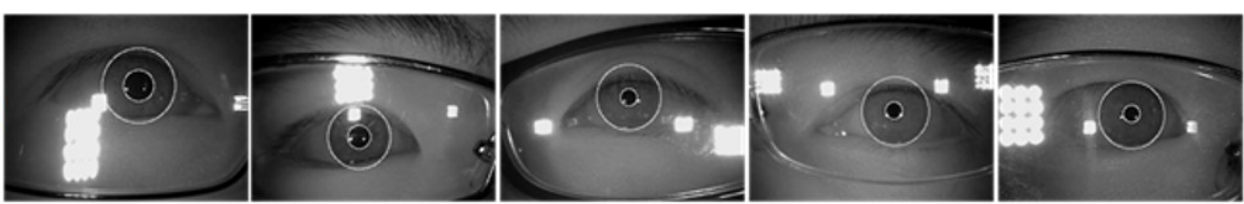

(b)
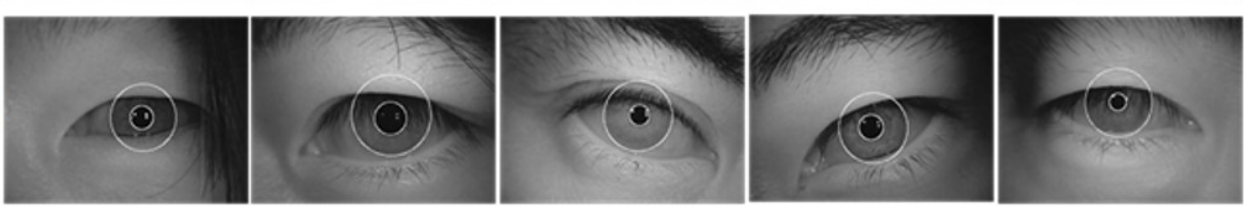

Figure 8. Accurately localized irises in the iris images from two CASIA databases [17]: (a) CITHV4; and (b) CILV3 


\subsection{Results and discussion}

The sample images with accurately localized irises by the proposed algorithm are shown in Figure 8 . The results of the proposed algorithm are summarized in Table 1.

Table 1. Experimental results of the proposed iris localization algorithm

\begin{tabular}{lcccc}
\hline Iris database & $\begin{array}{c}\text { Number of images taken } \\
\text { for testing }\left(\mathrm{N}_{\mathrm{t}}\right)\end{array}$ & $\begin{array}{c}\text { Number of correct iris localized } \\
\text { images }\left(\mathrm{N}_{\mathrm{i}}\right)\end{array}$ & $\begin{array}{c}\text { Accuracy }(\%)= \\
\left(\mathrm{N}_{\mathrm{i}} / \mathrm{N}_{\mathrm{t}}\right) \times 100\end{array}$ & $\begin{array}{c}\text { Average time cost per } \\
\text { image }(\mathrm{sec})\end{array}$ \\
\hline CITHV4* $(640 \times 480)$ & 5600 & 5583 & 99.7 & 0.92 \\
CILV3* $(640 \times 480)$ & 811 & 806 & 99.38 & 0.89 \\
\hline *CITHV4: CASIA-Iris-Thound $($ verion 4.0$) *$ CILV3 CASIA-Iris-Lmp $($ version 3.0) & &
\end{tabular}

*CITHV4: CASIA-Iris-Thousand (version 4.0); *CILV3: CASIA-Iris-Lamp (version 3.0)

The accuracy of the proposed algorithm is 100 percent almost. The accuracy of the circle detection in an image by the CHT depends on the amount of false edges the edge map of the image contains. Fewer the false edges higher would be the accuracy. The edge map used for the pupil boundary detection in the proposed algorithm contains very less false edges due to the intersection operation as discussed in the subsection 2.1.1. The use of the adaptive CHT for limbic boundary detection to counter the iris-occlusions by the eyelids and eyelashes is another cause for high accuracy.

Table 1 also shows the time performance results of the proposed method. The average time cost is reported in the table, as the time taken by the CHT for circle detection is directly proportional to the number of edge pixels in the edge map of the image. Fewer the false edges in the edge map of iris image, lesser will be the time cost. The average time cost per image was calculated by randomly choosing 500 images from each individual database. The MATLAB's timer functions 'tic' and 'toc' were used to know the execution time of a code that runs to localize irises in 500 images. The execution time obtained was then divided by 500 to find the average time cost per image.

\subsubsection{Comparison with other methods}

In our work, we also implemented the popular Wildes' [2] and Daugman's [1] methods for comparison with the proposed algorithm as the published results of these methods for CITHV4 and CILV3 databases are not available in the literature. Wildes' method [2] is based on the Canny edge detection plus CHT, whereas Daugman's method [1] uses the IDO as a circular edge detector. We applied both the approaches on the Gaussian smoothed iris images. The pupil was localized prior to the limbic boundary in both the methods.

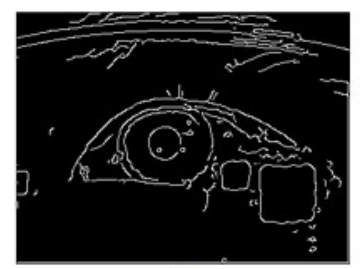

(a)

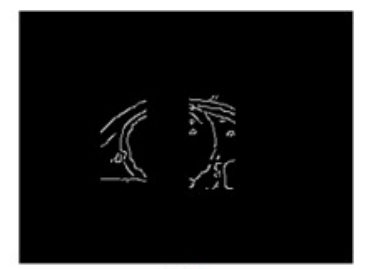

(b)

Canny edge detection with $\mathrm{T}=[0.034,0.085]$ and $\sigma$

$$
=1.0
$$

Figure 9. Edge maps of the iris image used in Fig.

2: (a) Edge map for pupil boundary detection; (b)

Edge map for limbic boundary detection

Table 2. Experimental results of iris localization

\begin{tabular}{lccc}
\multicolumn{4}{c}{ methods } \\
\hline Method & CITHV4 & CILV3 & MMUV1* \\
\hline Wildes [2] & 86.9 & 80.5 & 93.33 \\
Daugman [1] & 90.6 & 88.12 & 96.44 \\
Wildes [2]+Daugman's IDO [1] & 92.1 & 91.09 & 98 \\
Proposed & $\mathbf{9 9 . 7}$ & $\mathbf{9 9 . 3 8}$ & $\mathbf{9 9 . 5 5}$ \\
\hline
\end{tabular}

*MMUV1: Multimedia University, version 1.0

While using Wildes' method [2], we found that the limbic boundary's accuracy was coming very low due to the false edges of eyelids, eyelashes and pupil. So, we applied the CHT on the selected edge pixels in the Canny edge map; where the edge pixels were selected by placing two rectangles on left and right sides of the pupil as discussed in our proposed algorithm (Figure 5(c)). The edge maps used for Wildes' method [2] are shown in Figure 9.

While using Daugman's IDO [1] for the pupil detection, we observed that it is very sensitive to the reflection dots inside the pupil and gives wrong results. So, we removed these reflections during the pupil localization. The accuracy results of both the methods [1] and [2] are shown in Table 2. We also observed 
that localizing the pupil using Wildes' approach [2] and detecting the limbic boundary using Daugman's IDO [1] gives better results as compared to individual method as shown in Table 2.

Table 2 shows that both Wildes [2] and Daugman [1] methods give good accuracy for Multimedia University, version 1.0 (MMUV1) database [23] as it contains less noisy images, but their accuracy degrades for the noisy images of CITHV4 and CILV3. Wildes [2] gives less iris localization accuracy mainly due to the reflection spots in CITHV4 and too many false edges from the occlusions by eyelids and eyelashes in CILV3. These noises also reduce the accuracy of Daugman's IDO [1]. The average time cost per image obtained in Wildes' [2] and Daugman's [1] is $2.17 \mathrm{sec}$ and $2.45 \mathrm{sec}$ repectively, for the CITHV4 and CILV3 images of size $320 \times 240$ pixels. The proposed algorithm is more accurate and faster than Wildes [2] and Daugman [1] as it uses the optimal edge maps with very less false edges and the adaptive CHT for iris boundary detection also improves it further.

The comparison of the results of the proposed algorithm with the published results is shown in Table 3. The published methods included in the comparison are chosen on the basis that they used same databases for experimentation that we have taken. Moreover, Jan et al. [8],[9] show the highest accuracy among all the iris localization methods available in the literature for CITHV4 and CILV3 databases. The symbol -- in the table shows that the corresponding information was not found in the literature. The Table 3 shows that the proposed algorithm has the highest accuracy and lowest time cost per image, which has happened due to the proposed edge map and the adaptive CHT used for pupil and limbic boundary detection respectively, as compared to the other methods in the table. In the proposed method, the original iris image is scaled down to half size, which was also done in the Jan et al. methods [8],[9] to speed up the processing. The image resizing by a scaling factor, $\mathrm{s}=0.5$ not only reduces all the edge pixels to half in number, but also the number of radii taken in a CHT algorithm becomes half.

Table 3. Comparison with published iris localization results

\begin{tabular}{ccc}
\hline \multirow{2}{*}{ Method } & $\begin{array}{c}\text { Accuracy (\%) \& Average time cost per image (sec) } \\
\text { CITHV4 }\end{array}$ & CILV3 \\
\hline Jan et al. [8] & $99.5 \& 6.4$ & 98 \& 4.93 \\
Jan et al. [9] & $99.23 \& 3.4$ & $99.21 \& 3.35$ \\
Jan et al. [6] & -- & $99.05 \&--$ \\
Ibrahim et al.[24] & -- & $98.28 \&--$ \\
Proposed & $\mathbf{9 9 . 7 ~ \& ~ 0 . 9 2}$ & $\mathbf{9 9 . 3 8 ~ \& ~ 0 . 8 9}$ \\
\hline
\end{tabular}

\section{CONCLUSION}

The proposed iris localization method is tolerant to the non-ideal issues and noises in the iris images such as iris-occlusions by the eyelids and eyelashes, lighting reflections, non-uniform illumination, eyeglasses, low contrast and eyebrow hair. However, the experimental results show that the proposed method also improves iris localization in the images that do not have reflection spots and non-uniform illumination, but have mainly the iris-occlusions by the eyelids and eyelashes. The comparison with the famous Wildes' approach [2], which is based on Canny edge detection plus CHT, demonstrates that the introduction of new edge map for pupil boundary detection and adaptive CHT for limbic boundary detection make the proposed iris localization method more accurate and fast. The performance results of the proposed algorithm are much better than both the popular Daugman's [1] and Wildes' [2] approaches. The comparison with some recent published results for CASIA databases also shows that the proposed method has improved performance. The proposed algorithm can be used for the accurate iris segmentation in less constrained iris recognition systems.

\section{ACKNOWLEDGEMENTS}

We thankfully acknowledge Chinese Academy of Sciences' Institute of Automation (CASIA) for providing us the iris images. We also thank Multimedia University for providing MMU iris database.

\section{REFERENCES}

[1] J. Daugman, "High confidence visual recognition of persons by a test of statistical independence," IEEE Trans. Pattern Anal. Mach. Intell., vol/issue: 15(11), pp. 1148-1161, 1993.

[2] R. P. Wildes, "Iris recognition: an emerging biometric technology," Proc. IEEE, vol/issue: 85(9), pp. 1348-1363, 1997.

[3] L. Ma, et al., "Personal identification based on iris texture analysis," Pattern Anal. Mach. Intell. IEEE Trans., vol/issue: 25(12), pp. 1519-1533, 2003.

[4] K. W. Bowyer, et al., "Image understanding for iris biometrics: A survey," Comput. Vis. image Underst., vol/issue: 110(2), pp. 281-307, 2008. 
[5] F. Jan, et al., "A dynamic non-circular iris localization technique for non-ideal data," Computers and Electrical Engineering, vol/issue: 40(8), pp. 215-226, 2014.

[6] J. Daugman, "How iris recognition works," IEEE Trans. Circuits Syst. Video Technol., vol/issue: 14(1), pp. 21-30, 2004.

[7] N. Wang, et al., "Toward accurate localization and high recognition performance for noisy iris images," Multimedia Tools Appl., vol/issue: 71(3), pp. 1411-1430, 2014.

[8] F. Jan, et al., "Iris localization in frontal eye images for less constrained iris recognition systems," Digit. Signal Process. A Rev. J., vol/issue: 22(6), pp. 971-986, 2012.

[9] F. Jan, et al., "Reliable iris localization using Hough transform, histogram-bisection, and eccentricity," Signal Processing, vol/issue: 93(1), pp. 230-241, 2013.

[10] P. Li, et al., "Robust and accurate iris segmentation in very noisy iris images," Image Vis. Comput., vol/issue: 28(2), pp. 246-253, 2010.

[11] H. Proença, "Iris recognition: On the segmentation of degraded images acquired in the visible wavelength," IEEE Trans. Pattern Anal. Mach. Intell., vol/issue: 32(8), pp. 1502-1516, 2010.

[12] S. A. Sahmoud and I. S. Abuhaiba, "Efficient iris segmentation method in unconstrained environments," Pattern Recognit., vol/issue: 46(12), pp. 3174-3185, 2013.

[13] S. Khalighi, et al., "Iris recognition using robust localization and nonsubsampled contourlet based features," $J$. Sign. Process. Syst., vol/issue: 81(1), pp. 111-128, 2015.

[14] J. Zuo and N. A. Schmid, "On a methodology for robust segmentation of nonideal iris images," IEEE Trans. Syst. Man, Cybern. Part B Cybern., vol/issue: 40(3), pp. 703-718, 2010.

[15] K. M. I. Hasan and M. A. Amin, "Dual iris matching for biometric identification," Signal, Image and Video Processing, vol/issue: 8(8), pp. 1605-1611, 2014.

[16] T. Marciniak, et al., "Selection of parameters in iris recognition system," Multimed. Tools Appl., vol/issue: 68(1), pp. 193-208, 2014.

[17] CASIA Iris Image Database, 2010. http://biometrics.idealtest.org/.

[18] E. R. Davies, "Computer and machine vision: Theory, algorithms, practicalities," Academic Press, 2012.

[19] S. J. K. Pedersen, "Circular Hough transform," Aalborg University, Vision, Graphics and Interactive Systems, 2007.

[20] H. K. Yuen, et al., "Comparative study of Hough Transform methods for circle finding," Image Vis. Comput., vol/issue: 8(1), pp. 71-77, 1990.

[21] R. C. Gonzalez, et al., "Digital image processing using MATLAB," Gatesmark Publishing, 2009.

[22] A. Radman, et al., "Fast and reliable iris segmentation algorithm," IET Image Process., vol/issue: 7(1), pp. 42-49, 2013.

[23] MMU Iris Image Database, 2004. http://pesona. mmu.edu. my/ ccteo/.

[24] M. T. Ibrahim, et al., "Iris localization using local histogram and other image statistics," Opt. Lasers Eng., vol/issue: 50(5), pp. 645-654, 2012. 